\title{
Waveform Design for Massive MISO Downlink with Energy-Efficient Receivers Adopting 1-bit ADCs
}

Ahmet Gokceoglu, Emil Björnson, Erik G Larsson and Mikko Valkama

The self-archived postprint version of this conference article is available at Linköping University Institutional Repository (DiVA):

http:/ / urn.kb.se/ resolve?urn=urn:nbn:se:liu:diva-134225

N.B.: When citing this work, cite the original publication.

Gokceoglu, A., Björnson, E., Larsson, E. G, Valkama, M., (2016), Waveform Design for Massive MISO Downlink with Energy-Efficient Receivers Adopting 1-bit ADCs, 2016 IEEE INTERNATIONAL CONFERENCE ON COMMUNICATIONS (ICC). https:/ / doi.org/ 10.1109/ICC.2016.7510947

Original publication available at:

https:/ / doi.org/ 10.1109/ ICC.2016.7510947

Copyright: IEEE

http:// www.ieee.org/

(C)2016 IEEE. Personal use of this material is permitted. However, permission to reprint/ republish this material for advertising or promotional purposes or for creating new collective works for resale or redistribution to servers or lists, or to reuse any copyrighted component of this work in other works must be obtained from the IEEE. 


\title{
Waveform Design for Massive MISO Downlink with Energy-Efficient Receivers Adopting 1-bit ADCs
}

\author{
Ahmet Gokceoglu*, Emil Björnson ${ }^{\dagger}$, Erik G. Larsson ${ }^{\dagger}$, and Mikko Valkama* \\ ${ }^{*}$ Department of Electronics and Communications Engineering, Tampere University of Technology, Finland \\ e-mail: ahmet.gokceoglu@tut.fi,mikko.e.valkama@tut.fi \\ ${ }^{\dagger}$ Department of Electrical Engineering, Linköping University, Sweden \\ e-mail: emil.bjornson@liu.se, erik.g.larsson@liu.se
}

\begin{abstract}
In high-density low-bitrate Internet-of-Things (IoT) use case of $5 \mathrm{G}$ networks, the terminals and sensors are to be of extremely low-cost and low energy-consuming. Typically, the analog-to-digital converters (ADCs) dominate the power-budget of receiver chains, in particular if the quantization resolution is high. Hence, receiver architectures deploying 1-bit ADCs are of high interest towards realizing low-cost, high energy-efficiency device solutions. In this paper, we study the waveform design and optimization for a narrowband low-bitrate massive MISO downlink targeting to achieve rates higher than 1 bits/sec (per real-dimension) where the terminal receivers adopt only simple 1-bit quantization (per real-dimension ) with oversampling. In this respect, first we show that for a particular precoder structure, the overall link is equivalent to that of an AWGN SISO with controlled intersymbol interference (ISI). The filter design problem for generating the desired ISI in such SISO links has been studied in previous works, however, the only known method in literature is a computationally demanding brute force search method. As a novel contribution, we develop models and tools that elaborate on the conditions to be satisfied for unique detection and existence of solution for the filter coefficients. Then, as a concrete example, the developed models and tools are utilized to show that in the absence of noise, five-times oversampling is required for unique detection of 16-QAM input alphabet. Building on these findings, we then develop novel algorithms that can efficiently design the filter coefficients. Examples and simulations are provided to elaborate on filter coefficient design and optimization, and to illustrate good SER performance of the MISO link with 1-bit receiver even at SNRs down to $5 \mathrm{~dB}$.
\end{abstract}

\section{INTRODUCTION}

Internet-of-Things (IoT) is one of the use cases in upcoming $5 \mathrm{G}$ radio systems and refers to a network with enormous geographical density of low-bitrate low-cost devices or sensors, with applications e.g. in health care, remote metering, smart homes and smart vehicles [1]. In order to serve such massive numbers of devices, potentially up to 1 million devices per square-kilometer, substantial innovations in the radio access and networking solutions are needed. In general, 5G base stations (BS) are envisioned to deploy growing numbers of antennas to achieve extremely high beamforming

This work was supported by the Academy of Finland under the project 288670 "Massive MIMO: Advanced Antennas, Systems and Signal Processing at mm-Waves", the EU FP7 under ICT-619086 (MAMMOET) and the ELLIIT. and spatial multiplexing capabilities and gradually converge towards massive (large-scale) MIMO systems [2], [3]. In such networks, the cost- and energy-efficiency aspects must be carefully considered due to the large number of terminals as well as large number of BS antennas (and associated RF chains) if full digital beamforming is used. In general, the requirements to lower cost and energy consumption as well as demands for extended terminal battery-lifetimes call for novel communication schemes, transceiver architectures, and signal processing methods.

In this respect, [4] provides a detailed analysis and discussion on the uplink rates achievable by low-cost and highly energy-efficient massive MIMO BSs. As complementary works, [5] and [6] study the achievable MIMO downlink rates for high and low SNR regimes, respectively, where the receivers deploy highly energy-efficient 1-bit ADCs. Regarding the signal processing and waveform aspects of massive MIMO BSs, [7] proposes constant-envelope waveform design, enabling the use of energy-efficient non-linear power amplifiers. Another related work is [8] which considers hybrid digital and analog beamforming to decrease the number of BS RF chains and hence achieve higher energy-efficiency.

On the other hand, there are hardly any prior works that address the problem of waveform design and optimization to improve energy-efficiency at the terminals, especially in the IoT use case where receivers adopt ADCs with single-bit or low-number-of-bits. In a different context, [9], [10] studied the receiver energy-efficiency in high (multi-Gbps) bit-rate applications such as wireless backhauls and board-to-board communications. Since the energy consumption at receivers is typically dominated by power-hungry high-precision analogto-digital converters (ADCs), the authors proposed the use of 1-bit ADCs with oversampling. The core idea is to improve the overall energy efficiency while relying on additional signal processing to enable reliable decoding. It was shown that even with only 1-bit quantization (per real dimension) in the receiver, the system can achieve more than $1 \mathrm{bit} / \mathrm{channel}$ use capacity (per real dimension) with a proper oversampling factor and design of controlled inter-symbol interference (ISI). In [9], this controlled ISI is achieved by designing a 
pulse-shaping/ISI filter assuming a simplified model where there is no constraints from receive filtering and/or transmit bandwidth. Moreover, filter coefficients are optimized via a computationally demanding brute force search method which does not give any insight into the structure of this controlled ISI filter design such as the required oversampling rate and/or the relation between filter designs with different oversampling factors.

In this paper, we also build on this simplified model with no constraints from receiver filter and/or transmit bandwidth, however we have several novel contributions as follows. We first extend the narrowband (frequency-flat) SISO model in [9] to a narrowband massive MISO model, with specific focus on the IoT use case, i.e., transmission from a BS with a large antenna array to a simple receiver with a 1-bit ADC. Then, as another novel contribution, we elaborate in detail on the structure of the ISI filter design and optimization problem and also develop an algorithm to design the ISI filter coefficients, which substantially improves the computional efficiency compared to the brute force search method in [9].

The rest of this paper is organized as follows. The signal model for the SISO AWGN channel assumed in [9] is briefly reviewed in Section II whereas in Section III, it is shown that a massive MISO link with fading that deploys a particular transmit precoder is equivalent to such a SISO link under channel hardening. Then, in Section IV, the conditions are established for unique detection of input symbols in the absence of noise, assuming 1-bit ADC in the receiver, as well as for the existence of a unique solution to the underlying ISI filter coefficients. This is followed by devising an algorithm to design uniquely detectable and feasible codebook in Section $\mathrm{V}$, while another novel algorithm is proposed in Section VI to find the actual filter coefficients based on the designed codebook. The symbol detection aspects of the considered communication scheme is discussed in Section VII, while the results of numerical examples and computer simulations are presented in Section VIII. Finally, conclusions are drawn in Section IX.

\section{Signal Model For SISO AWGN Channels}

We consider a continuous time AWGN SISO model deploying IQ modulation/demodulation at the transmitter (TX) and the receiver (RX), respectively, as shown in Fig. 1 (a). The receiver is assumed to sample the signal with rate $F_{s}$ that is $N_{s} \in \mathbb{Z}^{+}$times higher than the symbol rate $R_{\text {sym }}$ and the quantization of the I and Q channel signals is done with a single-bit preserving only the sign of the received $I$ and $Q$ samples. The equivalent discrete-time baseband model is given in Fig. 1 (b). The complex symbol that is to be communicated at time $k$ is denoted as $\tilde{x}(k)=x^{I}(k)+j x^{Q}(k)$ and assumed to be from a square M-QAM constellation. There is identical real-valued (pulse-shape) ISI filtering on both $I$ and $Q$ branches and hence, the transmitted baseband complex vector reads

$$
\begin{aligned}
\tilde{\mathbf{s}}(k) & =\sum_{i=0}^{L-1} \mathbf{h}(i) x^{I}(k-i)+j \sum_{i=0}^{L-1} \mathbf{h}(i) x^{Q}(k-i) \\
& =\mathbf{s}^{I}(k)+j \mathbf{s}^{Q}(k)
\end{aligned}
$$

where $L$ is the memory length of the ISI and $\mathbf{h}(i)=$ $\left[h_{1}(i), \cdots, h_{N_{s}}(i)\right]^{T} \in \mathbb{R}^{N_{s} \times 1}$ is the impulse response vector of the ISI filter with oversampling factor of $N_{s}$ with respect to $R_{\text {sym }}$. On the receiver side, the noisy oversampled signal observation is denoted as $\mathbf{y}(k) \in \mathbb{C}^{N_{s} \times 1}$ which reads

$$
\tilde{\mathbf{y}}(k)=\underbrace{\left(\mathbf{s}^{I}(k)+\boldsymbol{\eta}^{I}(k)\right)}_{\mathbf{y}^{I}(k)}+j \underbrace{\left(\mathbf{s}^{Q}(k)+\boldsymbol{\eta}^{Q}(k)\right)}_{\mathbf{y}^{Q}(k)}
$$

where $\boldsymbol{\eta}^{I}(k)$ and $\boldsymbol{\eta}^{Q}(k)$ are being i.i.d. real-valued, zeromean AWGN vectors at the $I$ and $Q$ branches of the receiver, respectively. Then, the noisy over-sampled received signal is quantized with a 1-bit ADC on both $I$ and $Q$ branches, yielding

$$
\tilde{\mathbf{z}}(k)=\operatorname{sign}\left(\mathbf{y}^{I}(k)\right)+j \operatorname{sign}\left(\mathbf{y}^{Q}(k)\right)=\mathbf{z}^{I}(k)+j \mathbf{z}^{Q}(k)
$$

where $\operatorname{sign}\{$.$\} operates element-wise on the argument vector$ $\mathbf{v}$ such that $\operatorname{sign}\left\{\mathbf{v}_{i}\right\}=1$ if $\mathbf{v}_{i} \geq 0$, else $\operatorname{sign}\left\{\mathbf{v}_{i}\right\}=-1$.

\section{Signal Model for Narrowband Massive Miso FADING CHANNELS}

In our model, we assume that the massive MISO transmitter has $N_{t}$ transmit antennas and the complex fading coefficient between the $i^{\text {th }}$ transmit antenna and the 1-bit receiver is $\alpha_{i}$ which is an i.i.d. zero-mean circularly symmetric Gaussian random variable with variance $\sigma_{\alpha}^{2}$. The vector of fading coefficients is denoted as $\boldsymbol{\alpha}=\left[\alpha_{1}, \alpha_{2}, \cdots, \alpha_{N_{t}}\right]^{T}$. On transmitter side, the input symbols are spatially precoded prior to transmission yielding a complex baseband transmit matrix as

$$
\tilde{\mathbf{S}}(k)=\mathbf{P X}(k)
$$

where $\mathbf{X}(k)=\mathbf{I}_{N_{s}} \otimes[\tilde{x}(k), \tilde{x}(k-1)]^{T}$ with $\mathbf{I}_{N_{s}}$ being the identity matrix of dimension $N_{s}$ and $\otimes$ being the Kroneckerproduct. Now, denote $\mathbf{h}_{2 N_{s} \times 1}=\left[\mathbf{h}^{T}(0), \mathbf{h}^{T}(1)\right]^{T}$, and assume that the transmitter has perfect knowledge of the channel. The transmitter is then assumed to deploy a precoding matrix with the following decomposition extending the traditional maximum ratio transmission (MRT), written here as

$$
\mathbf{P}=\boldsymbol{\alpha}^{*} \mathbf{h}^{T}
$$

where $*$ denotes complex conjugation. Then, the transmit matrix expression in (4) can alternatively be written as

$$
\tilde{\mathbf{S}}(k)=\boldsymbol{\alpha}^{*}(x(k) \mathbf{h}(0)+x(k-1) \mathbf{h}(1))=\boldsymbol{\alpha}^{*} \tilde{\mathbf{s}}(k)
$$

Notice that (6) is simply the channel precoded version of the transmit vector given in (1). Following that, on receiver side, the complex oversampled signal vector before quantization reads

$$
\begin{aligned}
\tilde{\mathbf{y}}(k) & =\tilde{\mathbf{S}}^{T}(k) \boldsymbol{\alpha}+\tilde{\eta}(k)=\left(\boldsymbol{\alpha}^{H} \boldsymbol{\alpha}\right) \tilde{\mathbf{s}}(k)+\tilde{\eta}(k) \\
& \approx N_{t} \sigma_{\alpha}^{2} \tilde{\mathbf{s}}(k)+\tilde{\eta}(k)
\end{aligned}
$$




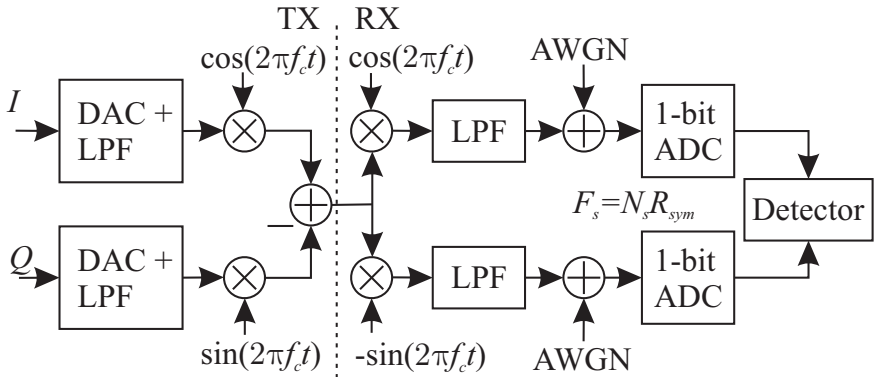

(a)

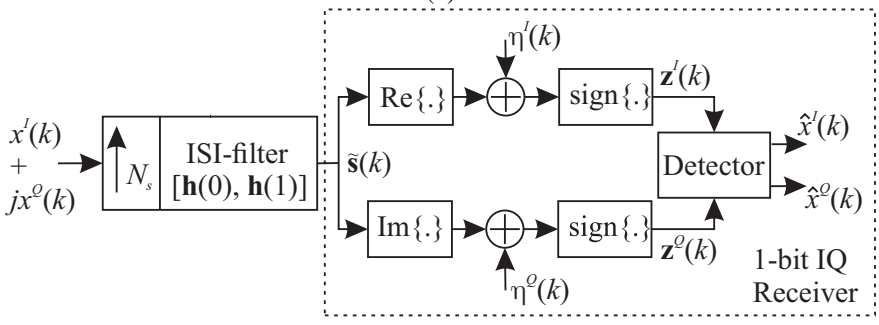

(b)

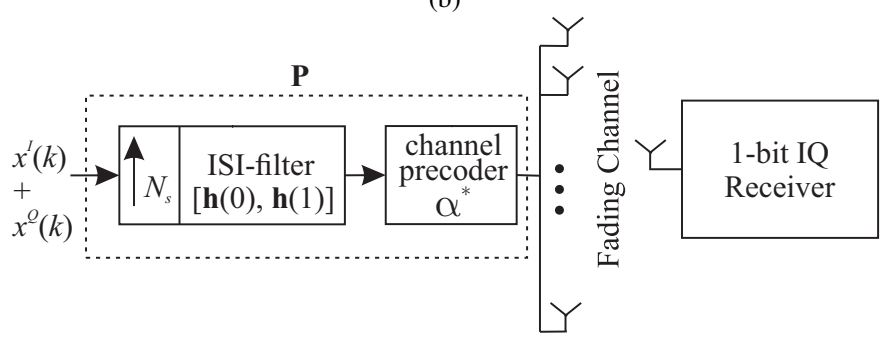

(c)

Fig. 1: Illustration of 1-bit receiver with oversampling (a) continuous-time AWGN SISO model (b) discrete-time baseband AWGN SISO model (c) discrete-time MISO model with fading

where superscript $H$ denotes Hermitian transpose. The approximation is due to channel hardening [11] and is tight for large $N_{t}$, e.g. $N_{t}>50$. Notice that the expression in (7) is equivalent to the AWGN SISO channel in (2) up to a scaling factor.

The form of the expression in (7) points out that the tools that are used for designing the ISI filter for AWGN SISO links can be used for massive MISO links with fading when the precoder has the specific decomposition given in (5). This is the main rationale in this paper where we will develop novel tools for the filter design and optimization problem building on the received signal model given by (2) and/or (7). Notice that the transmitter requires perfect channel-state information (CSI) in order to deploy the precoder given in (5). However, later in Section VIII, we will demonstrate that the SER performance under imperfect CSI is close to that of perfect CSI when a practical training sequence is used.

Notice also that the signal processing and statistical properties of additive noise are identical and independent in the $I$ and $Q$-branches. Hence, for the brevity of the notation, from this point on, we will drop the superscript and provide analysis and signal processing developments that can be applied at both branches, i.e., $x(k) \leftarrow x^{I}(k), \mathbf{s}(k) \leftarrow \mathbf{s}^{I}(k), \mathbf{z}(k) \leftarrow \mathbf{z}^{I}(k)$ and so on.

\section{Conditions For UniQue Detectability AND FEASIBILITY}

The challenge considering the system models in Sections II and III is to design the filter coefficients $\mathbf{h}$ such that the transmitted symbols can be uniquely detected in the absence of noise and also that reliable detection with low SER can be achieved when channel noise is present. In [9], due to computational complexity, the memory-length was restricted to $L=2$ which will also be the case in this work. However, for this special case of $L=2$, we will explore important properties and structures involved in the signal model. Based on these, we will present novel algorithms to be used in the filter design problem which have several significant advantages compared to the brute force method in [9]. Moreover, the discovered properties and developed tools for the case of $L=2$ is an important step towards the general case of $L>2$.

Now suppose $L=2$, and the current and previous symbols are $\{x(k), x(k-1)\}=\{1,-1\}$ or $\{3,-3\}$ where $x(k) \in$ $\{-3,-1,1,3\}$ (4-ASK per real-dimension). In the absence of noise, following the signal model from (1) to (3), the received quantized signal vectors $\mathbf{z}(k)$ would be identical for both transmissions causing ambiguity to the receiver. This ambiguity is resolved only if the receiver knows the previously transmitted symbol $x(k-1)$ as well. Thus, as also emphasized in [9], for unique detection, distinct symbols should be mapped to distinct quantized output-vectors once conditioned on a particular previous symbol. To differentiate from the noisy observations $\mathbf{z}(k)$, we will refer to such noiseless quantized output-vectors as conditional codewords and for $x(k)=a$ and $x(k-1)=u$ use the notation

$$
\mathbf{c}(a \mid u)=\operatorname{sign}\{a \mathbf{h}(0)+u \mathbf{h}(1)\}
$$

The two important remarks about these conditional codewords regarding unique detection are

$$
\begin{aligned}
& \text { U1) } \mathbf{c}(a \mid u) \neq \mathbf{c}(b \mid u) \text {, if } a \neq b \\
& \text { U2) } \mathbf{c}(a \mid u) \text { can be chosen same as } \mathbf{c}(b \mid w) \text {, if } u \neq w
\end{aligned}
$$

The condition in U1) guarantees unique detection; however it does not guarantee the existence of underlying filter coefficients $\mathbf{h}(0)$ and $\mathbf{h}(1)$. Hence, among the set of codewords that allow unique detection, we are actually interested in a subset where it is possible to find $\mathbf{h}(0)$ and $\mathbf{h}(1)$ that satisfy (8). We will refer to any such subset as feasible and as one of the novel contributions of this paper, explore how to find it next.

We define the symbol-ISI vector $\mathbf{x}^{(i, j)}=\left[x^{(i)}, x^{(j)}\right]$ where $x^{(i)}$ and $x^{(j)}$ are the amplitude levels to be transmitted at time $k$ and $k-1$, respectively. The symbol-ISI vectors are referred to as distinct if they satisfy $\mathbf{x}^{(i, j)} \neq \lambda \mathbf{x}^{(k, l)} \quad \forall \lambda \in \mathbb{R}^{+} \backslash\{1\}$. Then, we define a $D \times 2$ matrix containing all distinct symbolISI vectors as

$$
\mathbf{X}_{\text {dist }}=\left[\begin{array}{c}
\left(\mathbf{x}^{(1,1)}\right)^{T} \\
\vdots \\
\left(\mathbf{x}^{\left(i^{\prime}, j^{\prime}\right)}\right)^{T}
\end{array}\right]
$$




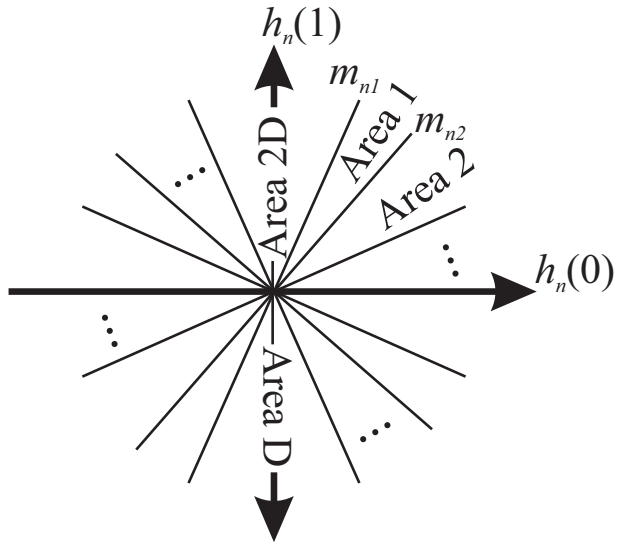

Fig. 2: Geometric interpretation of inequalities given by (12)-(13)

We also denote the corresponding $D \times N_{s}$ matrices whose rows contain all unquantized vectors and codewords as $\mathbf{S}_{\text {dist }}$ and $\mathbf{C}_{\text {dist }}$ respectively, which read

$$
\mathbf{S}_{\text {dist }}=\left[\begin{array}{c}
\left(\mathbf{s}^{(1,1)}\right)^{T} \\
\vdots \\
\left(\mathbf{s}^{\left(i^{\prime}, j^{\prime}\right)}\right)^{T}
\end{array}\right], \mathbf{C}_{\text {dist }}=\left[\begin{array}{c}
\left(\mathbf{c}\left(x^{(1)} \mid x^{(1)}\right)\right)^{T} \\
\vdots \\
\left(\mathbf{c}\left(x^{\left(i^{\prime}\right)} \mid x^{\left(j^{\prime}\right)}\right)\right)^{T}
\end{array}\right]
$$

where $\mathbf{s}^{(i, j)}=x^{(i)} \mathbf{h}(0)+x^{(j)} \mathbf{h}(1)$ and as given by (8) $\left.\mathbf{c}\left(x^{(i)} \mid x^{(j)}\right)\right)=\operatorname{sign}\left\{\mathbf{s}^{(i, j)}\right\}$. The matrix $\mathbf{C}_{\text {dist }}$ will be referred to as the codebook. Denoting $m_{n}=-\frac{x^{(i)}}{x^{(j)}}$, we have

$$
\mathbf{c}_{n}\left(x^{(i)} \mid x^{(j)}\right)= \begin{cases}1, & \text { if } h_{n}(1)>m_{n} h_{n}(0) \\ -1, & \text { if } h_{n}(1)<m_{n} h_{n}(0)\end{cases}
$$

Hence, the $n^{t h}$ column of $\mathbf{C}_{\text {dist }}$ dictates $D$ distinct linear inequalities to be satisfied which are geometrically visualized in Fig. 2. The 2-dimensional $h_{n}(1)-h_{n}(0)$ plane is divided by $D$ distinct lines passing through the origin and either the area under or above the line is taken according to the direction of the inequality. Thus, a solution to filter coefficients $h_{n}(1)$ and $h_{n}(0)$ exists if and only if the intersection of these $D$ areas is non-zero. Now suppose that, a row permutation is applied to the codebook matrix such that $\breve{\mathbf{C}}_{\text {dist }}=\mathbf{P}_{r} \mathbf{C}_{\text {dist }}$, and for a particular column, the slopes corresponding to each row have a decreasing order $m_{n_{1}}>m_{n_{2}}>\cdots>m_{n_{D}}\left(m_{n_{i}}\right.$ : slope of $i^{\prime}$ th row). If the solution to $h_{n}(1)$ and $h_{n}(0)$ lies in one of the areas $i \in A_{D}=\{1, \cdots, D\}$ or $i \in A_{2 D}=\{D+1, \cdots, 2 D\}$, then the corresponding inequalities should satisfy

$$
\begin{aligned}
& \text { Area } i \in A_{D}: h_{n}(1)\left\{\begin{array}{l}
<m_{n_{j}} h_{n}(0), \text { for } j \leq i \\
>m_{n_{j}} h_{n}(0), \text { for } j>i
\end{array}\right. \\
& \text { Area } i \in A_{2 D}: h_{n}(1)\left\{\begin{array}{l}
>m_{n_{j}} h_{n}(0), \text { for } j \leq i-D \\
<m_{n_{j}} h_{n}(0), \text { for } j>i-D
\end{array}\right.
\end{aligned}
$$

Plugging (13) into (12), the $n^{\text {th }}$ column corresponding to Area $i$ is $\left(\breve{\mathbf{C}}_{\text {dist }}\right)_{:, n}=\mathbf{v}(i)$ where

$$
\mathbf{v}(i)=\left\{\begin{array}{l}
{\left[-\mathbf{1}_{i}^{T}, \mathbf{1}_{D-i}^{T}\right]^{T}, \text { if } 1<i \leq D} \\
{\left[\mathbf{1}_{i-D}^{T},-\mathbf{1}_{2 D-i}^{T}\right]^{T}, \text { if } D<i \leq 2 D}
\end{array}\right.
$$

In summary, (9), (U1) and (14) define the necessary and sufficient conditions for rows and columns of codebooks that are uniquely detectable and feasible.

\section{Proposed Algorithm to Generate Uniquely Detectable and Feasible Codebooks}

We can now devise an algorithm that generates a codebook with the row and column structures imposed by (9), (U1) and (14) and targets at minimum number of columns, i.e., minimum oversampling rate. The main rationale is to start with an empty matrix and at each iteration append a proper vector $\mathbf{v}(i)$ that differentiates the codewords in the desired rows and upon completion of the procedure satisfy $\mathrm{U} 1$.

For a given $\mathbf{v}(i)$, if we define $r^{\text {flip }}$ as the row number where there is a sign change, then there are two properties inherent to the process of appending a new column to the iterated codebook

P1) $r^{\text {flip }}$ differentiates all rows $r_{1}: r^{\text {flip }}-1$ from rows $r^{\text {flip }}$ : $r_{D}$

P2) To differentiate row $r_{i}$ from rows $r_{j}, \cdots, r_{m}$ satisfying $r_{i}<r_{j}<\cdots<r_{m}$, then $r^{\text {flip }}$ should satisfy $r_{i}<r^{\text {flip }}<r_{j}-1$. We will denote smallest row to be differentiated $\left(r_{j}\right)$ as $r_{i}^{\mathrm{min}}$.

In order to construct a codebook with as few columns as small as possible, at each iteration of the algorithm, a new vector $\mathbf{v}(i)$ will be appended that differentiates maximum number of rows. The algorithm needs to know $D$ (row number of $\mathbf{X}_{\text {dist }}$ ) and $r_{i}^{\min } \forall i \in\{1, \cdots, D\}$ which can be easily obtained as follows. For a given modulation order (per real dimension), one can generate all possible symbol-ISI vectors $\mathbf{x}^{(i, j)}$ and construct $\mathbf{X}_{\text {dist }}$ (10) by keeping only one of the vectors that are positive multiples of each other after which $D$ is simply the row number of $\mathbf{X}_{\text {dist }}$. Then, if $\mathbf{x}^{(i, j)}=\left[x^{(i)}, x^{(j)}\right]$ is the $i$ 'th row of $\mathbf{X}_{\text {dist }}, \mathbf{c}\left(x^{(i)}, x^{(j)}\right)$ is the $i$ 'th row of $\mathbf{C}_{\text {dist }}$ for which $r_{i}^{\min }$ can be obtained via U1. We also define vectors $(\mathbf{d})_{D \times 1}$ and $\left(\mathbf{d}^{\#}\right)_{K \times 1}$ such that

$$
\begin{aligned}
\mathbf{d}_{i} & = \begin{cases}1, & \text { if } r_{i} \text { differentiated from } r_{i}^{\min } \\
0, & \text { otherwise }\end{cases} \\
\mathbf{d}_{j}^{\#} & =\text { number of rows differentiated by } \mathbf{v}(j)
\end{aligned}
$$

We initialize, $k=0$, d to all zero vector and $\breve{\mathbf{C}}_{\text {dist }}$ to an empty matrix. Then the full algorithm is: 
Algorithm 1: Unique and Feasible Codebook Generation

step 1: Initialize $\mathbf{d}^{\#}$ to all zero vector, go to step 2.

step 2: Set $k=k+1$, get $\mathbf{v}(k)$, find corresponding $r^{f l i p}$, go to step 3 .

step 3: For $l=1: r^{f l i p}$, if $r_{l}^{\min } \geq r^{f l i p}$ and $\mathbf{d}_{l} \neq 1$, set $\mathbf{d}_{k}^{\#}=\mathbf{d}_{k}^{\#}+1$. If $k=K$, go to step 4, else to step 2 .

step 4: Get the row $r^{\max }$ of $\mathbf{d}^{\#}$ which has max. value. Append $\mathbf{v}\left(r^{\max }\right)$ as a new column to $\breve{\mathbf{C}}_{\text {dist }}$. Find $r^{\text {flip }}$ of $\mathbf{v}\left(r^{\max }\right)$. For $m=1: r^{f l i p}$, if $r_{l}^{\min } \geq r^{f l i p}$ and $\mathbf{d}_{l} \neq 1$, set $\mathbf{d}_{l}=1$. If $\mathbf{d}$ is all $1 \mathrm{~s}$ vector, obtain $\mathbf{C}_{d i s t}=$ $\mathbf{P}_{r}^{T} \breve{\mathbf{C}}_{d i s t}$ and finish the procedure, else set $k=0$ and go to step 1 .

Note that the number of columns of $\breve{\mathbf{C}}_{d i s t}$ obtained via the above algorithm tells what should be the oversampling rate $N_{s}$ for unique and feasible detection. However, it might be desired to have an oversampling rate higher than $N_{s}$ since longer codewords provide better SER due to increased distance between the words. In that case, it is sufficient to append any column vector from $V_{C}$ to the existing $\breve{\mathbf{C}}_{\text {dist }}$, however, the choice affects whether the SER performance will be better or worse than the case with lower oversampling. Hence, it is not straightforward to conclude that higher oversampling yields better SER performance, and this will be elaborated more in Section VIII.

\section{Vi. Proposed Optimization of Filter Coefficients}

Given the codebook $\mathbf{C}_{d i s t}$ designed by the above algorithm, a region in $\mathbb{R}^{2}$ is defined where the solution to $h_{n}(1)$ and $h_{n}(0)$ exists. In this section we find the actual values of these coefficients within the specified region based on specific optimization constraints. We use a similar criterion as in [9] which maximizes the so-called minimum unquantized filter output level $\gamma$ such that $\left|\mathbf{s}_{n}^{(i, j)}\right|>\gamma \forall i, j, n$. Higher values of $\gamma$ provide better protection against errors since it will be more difficult for the noise to alter the sign of any element of the codeword. Together with a squared norm constraint on the filter coefficients, the overall optimization problem for $h_{n}(1)$ and $h_{n}(0)$ can be stated as

$$
\begin{aligned}
\operatorname{maximize} & \gamma_{n} \\
\text { subject to: } \operatorname{diag}\left\{\left(\mathbf{C}_{d i s t}\right)_{:, n}\right\} & \left(\mathbf{S}_{d i s t}\right)_{:, n} \succeq \gamma_{n} \mathbf{1}_{D} \\
& \left\|\left[h_{n}(0), h_{n}(1)\right]\right\|^{2}=c
\end{aligned}
$$

where $c$ is a constant, $\succeq$ is an elementwise comparison and $\operatorname{diag}\{$.$\} returns a diagonal matrix whose diagonal entries are$ given by the argument vector. Defining $\mathbf{a}=[0,0,-1]^{T}, \mathbf{r}=$ $\left[h_{n}(0), h_{n}(1), \gamma_{n}\right]^{T}, \mathbf{B}=\left[\operatorname{diag}\left\{\left(\mathbf{C}_{\text {dist }}\right)_{:, n}\right\} \mathbf{X}_{\text {dist }}, \mathbf{1}\right]$, the above optimization problem can be brought to standard convex form (as given similarly in [9]),

$$
\begin{array}{ll}
\text { minimize } & \mathbf{a}^{T} \mathbf{r} \\
\text { subject to: } & -\mathbf{B r} \prec \mathbf{0} \text { and } \mathbf{r}^{T}\left[\begin{array}{ll}
\mathbf{I} & \mathbf{0} \\
\mathbf{0} & 0
\end{array}\right] \mathbf{r} \leq c
\end{array}
$$

As argued in [9], (16) can be solved via available convex optimization tools such as CVX [12]. However, there are two major differences between the formulation in (16) and the one given in [9].

The first difference is that in [9], the column dimension (oversampling factor $N_{s}$ ) and the entries of the codebook $\mathbf{C}_{d i s t}$ should be guessed. The optimization problem is solved for each guess and a final maximation is applied among all solutions. Even for small $N_{s}$ and low order modulation, the entire search space of codebooks can easily be over $10^{N}$ with $N \gg 6$ (a subspace of $10^{6}$ codebooks is considered in [9]). Such a brute force method is completely avoided in our formulation by discovering the structure of unique and feasible codebooks and hence generating $\mathbf{C}_{\text {dist }}$ by the Algorithm 1 in Section $V$.

The second difference is that, we attempt to solve for $h_{n}(1)$ and $h_{n}(0)$ for each $n$ separately whereas the formulation in [9] tries to solve for all coefficients of $\mathbf{h}(1)$ and $\mathbf{h}(0)$ at once. Hence, opposed to a large minimization problem of $2 N_{s}$ unknowns, we attempt to solve for a substantially smaller minimization problem with only 2 unknowns. Considering that typically $N_{s}>5$, for any practical minimization algorithm, our formulation is thus much easier to handle.

Now suppose that we have solved for $h_{n}(1)$ and $h_{n}(0)$ for all $n$, and obtained a minimum unquantized filter output level vector $\gamma=\left[\gamma_{1}, \cdots, \gamma_{N_{s}}\right]$. Note that the SER in the receiver is mostly determined by the minimum element of this vector. In fact, the large maximization problem in [9] tries to maximize the minimum element of this vector. Hence, our formulation can inherit the same property with an additional yet simple weighting step. Assume that the filter coefficients obtained from (16) are weighted to obtain new coefficients as $h_{n}(1) \leftarrow w_{n} h_{n}(1)$ and $h_{n}(0) \leftarrow w_{n} h_{n}(0)$ such that $\gamma_{n} \leftarrow w_{n} \gamma_{n}$. Now, $w_{n}$ 's can be chosen such that the minimum element of the new $\gamma$ are all equal, i.e., $w_{1} \gamma_{1}=\cdots=w_{N_{s}} \gamma_{N_{s}}$. However, typically there is a constraint on the squared norm of the overall filter which according to constraint in (15) can be written as $\left\|\left[\mathbf{h}^{T}(0), \mathbf{h}^{T}(1)\right]\right\|^{2}=c N_{s}=c \sum_{n} w_{n}^{2}$. From these two conditions, we find the weights to be of the form

$$
w_{1}=\sqrt{\frac{N_{s}}{\sum_{i=1}^{N_{s}}\left(\frac{\gamma_{1}}{\gamma_{i}}\right)^{2}}}, \quad w_{i}=w_{1} \frac{\gamma_{1}}{\gamma_{i}}
$$

The overall design of the ISI filter coefficients is summarized in the algorithm given below:

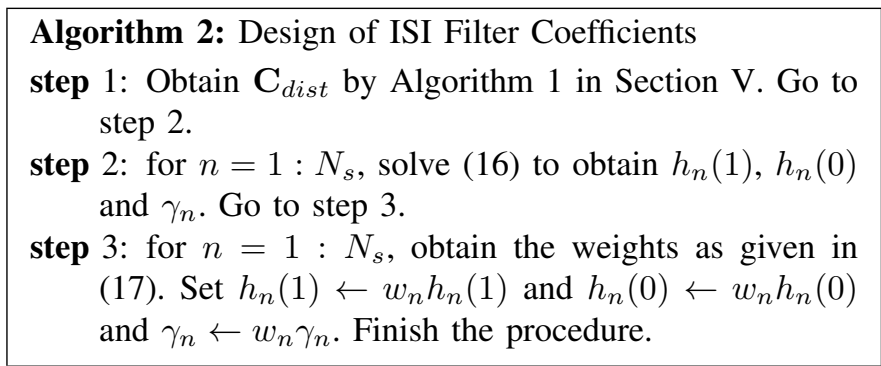

Now, assume that this previous design is desired to be updated for a higher oversampling rate as mentioned in the last 
paragraph of Section V. Also assume that the new codebook has the first $N_{s}$ columns the same as the old one whereas there are $N_{s}^{\prime}$ additional columns. In that case, the above algorithm can be run only for these new columns for which filter coefficients and minimum unquantized filter output levels are obtained. The new weights are calculated again using (17) and the step 3 of the algorithm is applied with an additional scale factor of $\kappa=\sqrt{N_{s} /\left(N_{s}+N_{s}^{\prime}\right)}$ due to the constraint on $\left\|\left[\mathbf{h}^{T}(0), \mathbf{h}^{T}(1)\right]\right\|^{2}$. Hence we have $h_{n}(i) \leftarrow \kappa w_{n} h_{n}(i)$ and $\gamma_{n} \leftarrow \kappa w_{n} \gamma_{n}$.

\section{Detection Aspects}

In general, the above transmission scheme or waveform design where controlled ISI is introduced is analogous to a convolutional code with the memory length limited to one previous symbol only. Hence, the number of states is equal to the input alphabet size per real-dimension. The Viterbi algorithm can then be used as a maximum-likelihood detector. However, since one of the main motivations of deploying 1bit ADCs is to have a simple receiver, we focus on alternative suboptimal symbol detector.

Given that the previously detected symbol is $\hat{\mathbf{x}}(k-$ 1) $=x^{(j)}$, the receiver calculates the Hamming distance between the current noisy quantized observation $\mathbf{z}(k)$ and $\mathbf{c}\left(x^{(i)} \mid x^{(j)}\right)$ for all $i$ and decides on the current symbol as $\hat{\mathbf{x}}(k)=\arg \min \left\{\operatorname{Hamming}\left(\mathbf{z}(k), \mathbf{c}\left(x^{(i)} \mid x^{(j)}\right)\right)\right\}$ where

$\operatorname{Hamming}(\mathbf{u}, \mathbf{v})^{x^{(i)}}=d$ if vectors $\mathbf{u}$ and $\mathbf{v}$ differ in $d$ positions. This very simple processing is used as the detection method in the simulations presented in the next section.

\section{EXAMPLES, Simulations AND Discussions}

In this section, we provide examples of the developed algorithms in designing and optimizing the ISI filter coefficients and present the corresponding achievable SER performance. For this purpose, we consider the complex input alphabet to be 16-QAM which corresponds to a 4-ASK scheme per real dimension, i.e., $x^{(i)} \in\{-3,-1,1,3\}$. The size of the distinct symbol-ISI pair set is found to be $D=6$ and the codebook obtained by the Algorithm 1 in Section V is as follows:

$$
\mathbf{C}_{\text {dist }}=\left[\begin{array}{c}
\mathbf{c}(1 \mid 3) \\
\mathbf{c}(-1 \mid 3) \\
\mathbf{c}(3 \mid 1) \\
\mathbf{c}(1 \mid 1) \\
\mathbf{c}(-1 \mid 1) \\
\mathbf{c}(-3 \mid 1)
\end{array}\right]=\left[\begin{array}{ccccc}
1 & 1 & -1 & -1 & -1 \\
1 & 1 & 1 & -1 & -1 \\
-1 & -1 & -1 & -1 & -1 \\
1 & -1 & -1 & -1 & -1 \\
1 & 1 & 1 & 1 & -1 \\
1 & 1 & 1 & 1 & 1
\end{array}\right]
$$

Hence, the oversampling factor is $N_{s}=5$. Note that $\mathbf{C}_{\text {dist }}$ given in (18) provides sufficient information for the whole codebook, the remaining codewords that are not given can be trivally derived from codewords in (18), e.g. $\mathbf{c}(-3 \mid-1)=$ $-\mathbf{c}(3 \mid 1), \mathbf{c}(3 \mid 3)=\mathbf{c}(1 \mid 1)$ and similarly for other codewords. Then, by applying the Algorithm 2 in Section VI, the filter coefficients are found to be

$$
\mathbf{H}=\left[\begin{array}{l}
\mathbf{h}^{T}(0) \\
\mathbf{h}^{T}(1)
\end{array}\right]=\left[\begin{array}{ccccc}
0.2 & 0.4 & 0.2 & 0.4 & 0.2 \\
0.4 & 0.2 & 0 & -0.2 & -0.4
\end{array}\right]
$$

Note that these coefficients are practically the same as the ones given in [9], however we now have a much more computationally efficient filter design. Moreover, we have shown that these coefficients are optimal in MISO fading case in addition to their optimality in SISO AWGN as claimed in [9].

Using the designed filter above, in Fig. 3, SER vs. SNR curves are plotted for SISO AWGN and MISO block fading channels. The SNR is defined as $\mathrm{SNR} \doteq \frac{E\left[\operatorname{trace}\left\{\tilde{\mathbf{S}}(k) \tilde{\mathbf{S}}^{H}(k)\right\}\right]}{E\left[\operatorname{trace}\left\{\boldsymbol{\eta}(k) \boldsymbol{\eta}^{H}(k)\right\}\right]}=$ $\frac{N_{t} \sigma_{\alpha}^{2} \sigma_{x}^{2}\|\mathbf{h}\|^{2}}{N_{s} \sigma_{\eta}^{2}}$. In the fading case, the channel is stationary during a coherence interval of 200 symbols and 5 uplink training symbols are used for least-squares based estimation of the channel coefficients at the transmitter, used then for downlink precoding, i.e., a TDD scheme is applied. Dotted lines correspond to perfect CSI whereas channel is estimated via uplink pilots for the solid lines. We set a moderate level of $S N R=5 \mathrm{~dB}$ during the channel estimation stage. In Section III, the signal model was based on perfect CSI assumption after which we showed the equivalence of SISO and MISO models. Here, the dotted lines corresponding to perfect CSI and hence following the assumed signal model demonstrate very good SER behavior improving with increasing SNR (for fixed $N_{t}$ ) and with increasing $N_{t}$ (for fixed SNR). In addition, the solid lines corresponding to more practical imperfect CSI scenario show similar SER behavior and the performances are close in the low-to-moderate $\mathrm{SNR}$ region, e.g. up-to $\mathrm{SNR}=5$ $\mathrm{dB}$ whereas a similar performance is also observerd with estimated channel. If the number of antennas is increased to $N_{t}=100$, than the SER improvement under imperfect CSI is even more pronounced in the same SNR interval, decreasing from $3 \times 10^{-1}$ to $5 \times 10^{-3}$. Also, it is seen that the SER performance of 1-bit quantized AWGN SISO link is rather poor compared to the performance of 1-bit quantized fading MISO link with increasing number of antennas.

In the next example, illustrated in Fig. 4, the aim is to demonstrate how the SER performance can be improved by increasing the oversampling rate (here only hard-symbol detector is presented). As discussed in the last paragraph of Section V, the designed codebook in (18) will be updated by appending a new $\mathbf{v}(i)$ given in (14) and the previous filter design will be updated as discussed in the last paragraph of Section VI. It is calculated that, $\mathbf{v}(3)$ and $\mathbf{v}(9)$ yield the filter coefficients that provide highest $\gamma$. Moreover, except for the vectors $\mathbf{v}(6)$ and $\mathbf{v}(12)$, appending by any other vector would increase the Hamming distance between the codewords, however, the increase is the highest with $\mathbf{v}(3)$ and $\mathbf{v}(9)$. Hence, we expect to see the highest increase in SER upon appending these ones to the codebook and solving for filter coefficients. This behavior can be clearly seen in Fig. 4 where there is improvement in SER when $N_{s}$ is increased from 5 to 7 with the use of $\mathbf{v}(3)$ two times. On the other hand, the SER has not changed when the vector $\mathbf{v}(4)$ is used instead which is due to the scaling with $\kappa$ elaborated in the last paragraph of Section VI. We can conclude that the improvement due to increase in Hamming distance, is canceled by the degradation 


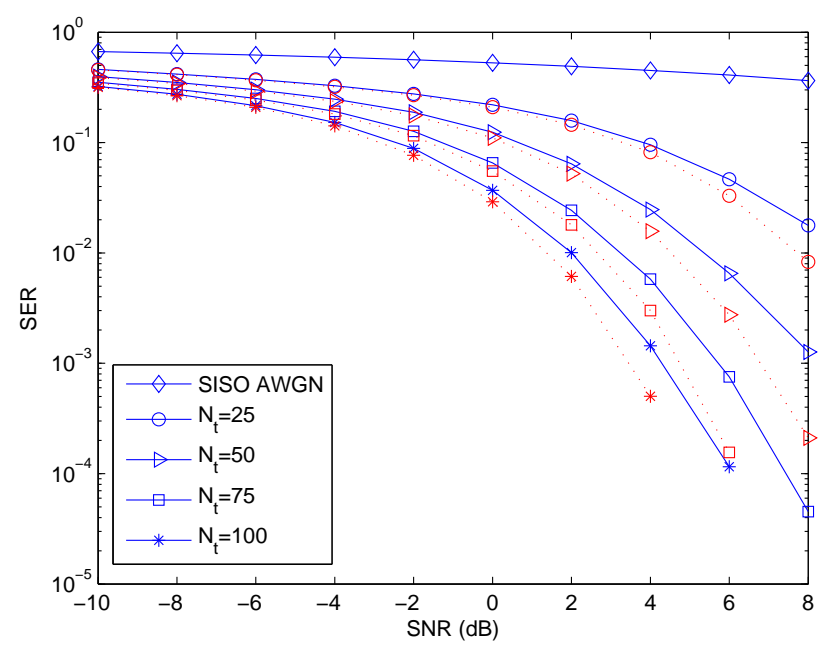

Fig. 3: SER vs. SNR for oversampling factor of $N_{s}=5$ and indicated values of $N_{t}$. Used modulation is 16-QAM. Dotted and solid lines correspond to perfect and imperfect CSI, respectively. The DL CSI is estimated from UL reference symbols in the imperfect CSI case.

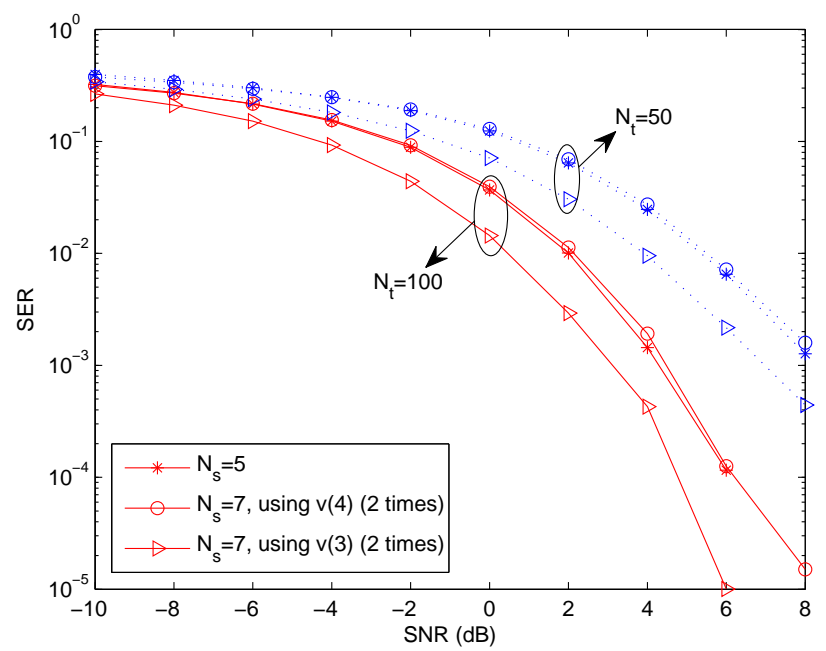

Fig. 4: SER vs. SNR for indicated values of oversampling factor $N_{s}$ and $N_{t}$. The vectors appended to extend previous $\mathbf{C}_{d i s t}$ are indicated in the legend. Used modulation is 16-QAM. Only the imperfect CSI case is shown.

effect of reduced $\gamma$.

\section{CONCLUSION}

In this paper, we considered the problem of waveform and transmit ISI filter design and optimization for communicating towards receivers deploying 1-bit ADCs and oversampling. We have shown that the received signal models for AWGN SISO and massive MISO link with narrowband fading are equivalent for a particular decomposition of the precoder in MISO transmitters together with the assumption of channel hardening. We developed and proposed novel tools and algorithms that elaborate on the structure of the problem and that can efficiently design the filter coefficients, opposed to the computationally demanding brute force search method considered in the existing literature. Another important advantage of the developed tools is that any previous design for a specific oversampling rate $N_{s}$ can be reused in the design for an oversampling rate $N_{s}^{\prime}>N_{s}$ which was not possible with the brute force method that requires the design to be redone from the beginning. The obtained results indicate that the deployment of 1-bit receivers is feasible in massive MISO transmission, under properly optimized transmit precoder and waveform design, and thus very simple sensor receivers can potentially be adopted in IoT use cases in the emerging $5 \mathrm{G}$ networks. Our future work will focus on extending the study to more practical ISI lengths of $L>2$, mainly imposed by receiver filter and to multi-user spatial multiplexing scenarios with multiple 1-bit receivers being served simultaneously.

\section{REFERENCES}

[1] A. Gupta and R. Jha, "A Survey of 5g Network: Architecture and Emerging Technologies," IEEE Access, vol. 3, pp. 1206-1232, 2015.

[2] F. Rusek, D. Persson, B. K. Lau, E. Larsson, T. Marzetta, O. Edfors, and F. Tufvesson, "Scaling Up MIMO: Opportunities and Challenges with Very Large Arrays," IEEE Signal Processing Magazine, vol. 30, pp. 40-60, Jan. 2013.

[3] H. Q. Ngo, E. Larsson, and T. Marzetta, "Energy and Spectral Efficiency of Very Large Multiuser MIMO Systems," IEEE Transactions on Communications, vol. 61, pp. 1436-1449, Apr. 2013.

[4] E. Bjornson, M. Matthaiou, and M. Debbah, "Massive MIMO with Non-Ideal Arbitrary Arrays: Hardware Scaling Laws and Circuit-Aware Design," IEEE Transactions on Wireless Communications, vol. 14, pp. 4353-4368, Aug. 2015

[5] J. Mo and R. Heath, "Capacity Analysis of One-Bit Quantized MIMO Systems With Transmitter Channel State Information," IEEE Transactions on Signal Processing, vol. 63, pp. 5498-5512, Oct. 2015.

[6] A. Mezghani and J. Nossek, "On Ultra-Wideband MIMO Systems with 1-bit Quantized Outputs: Performance Analysis and Input Optimization," in IEEE International Symposium on Information Theory, 2007. ISIT 2007, pp. 1286-1289, June 2007.

[7] S. Mohammed and E. Larsson, "Constant-Envelope Multi-User Precoding for Frequency-Selective Massive MIMO Systems," IEEE Wireless Communications Letters, vol. 2, pp. 547-550, Oct. 2013.

[8] L. Dai, X. Gao, J. Quan, S. Han, and C.-L. I, "Near-optimal hybrid analog and digital precoding for downlink mmWave massive MIMO systems," pp. 1334-1339, June 2015.

[9] L. Landau, S. Krone, and G. Fettweis, "Intersymbol-Interference Design for Maximum Information Rates with 1-Bit Quantization and Oversampling at the Receiver," in Proceedings of 2013 9th International ITG Conference on Systems, Communication and Coding (SCC), pp. 1-6, Jan. 2013.

[10] S. Krone and G. Fettweis, "Communications with 1-bit quantization and oversampling at the receiver: Benefiting from inter-symbol-interference," in 2012 IEEE 23rd International Symposium on Personal Indoor and Mobile Radio Communications (PIMRC), pp. 2408-2413, Sept. 2012.

[11] B. Hochwald, T. Marzetta, and V. Tarokh, "Multiple-antenna channel hardening and its implications for rate feedback and scheduling," IEEE Transactions on Information Theory, vol. 50, Sept. 2004.

[12] M. Grant and S. Boyd, "Cvx: Matlab/ software for disciplined programming, version 1.21," http://cvxr.com/cvx, Jan. 2011. 